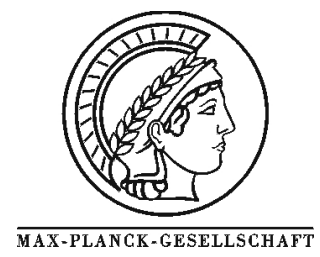

\title{
Characterization of Thin Films containing Zirconium, Oxygen, and Sulfur by Scanning Electron and Atomic Force Microscopy
}

\author{
A. Fischer ${ }^{1}$, F.C. Jentoft ${ }^{1 *}$, G. Weinberg ${ }^{1}$, R. Schlögl ${ }^{1}$, \\ T.P. Niesen ${ }^{2}$, J. Bill², F. Aldinger ${ }^{2}$, M.R. De Guire ${ }^{3}$, M. Rühle ${ }^{4}$ \\ ${ }^{1}$ Department of Inorganic Chemistry, Fritz-Haber-Institute of the MPG, Faradayweg 4-6, 14195 Berlin, Germany \\ ${ }^{2}$ Max-Planck-Institut für Metallforschung und Institut für Nichtmetallische Anorganische Materialien, \\ Pulvermetallurgisches Laboratorium, Heisenbergstraße 5, 70569 Stuttgart, Germany \\ ${ }^{3}$ Department of Materials Science and Engineering, Case Western Reserve University, 10900 Euclid Avenue, \\ Cleveland, Ohio 44106-7204, USA \\ ${ }^{4}$ Max-Planck-Institut für Metallforschung, Seestraße 92, 70174 Stuttgart, Germany
}

${ }^{*}$ Corresponding author: e-mail jentoft@fhi-berlin.mpg.de

Received 01 February 1999, accepted 24 May 1999

\begin{abstract}
Oxidic zirconium films prepared by chemical deposition from aqueous medium on sulfonic acid terminated self-assembled monolayers attached to an oxidized silicon surface were investigated with scanning electron microscopy and atomic force microscopy. Bulk precipitate forms in the $4 \mathrm{mM} \mathrm{Zr}\left(\mathrm{SO}_{4}\right)_{2} * 4 \mathrm{H}_{2} \mathrm{O}, 0.4 \mathrm{~N} \mathrm{HCl}$ deposition medium at $343 \mathrm{~K}$ after approximately $30 \mathrm{~min}$. Precipitate particles (200 nm and larger) were found embedded into the oxidic zirconium film and adsorbed on top of the film; they could be washed off but patches of the film were removed. Working with unstable deposition solutions, in which homogeneous nucleation occurs, leads to preparation-inherent flaws in the film.
\end{abstract}

\section{Introduction}

Zirconia coatings are of technical importance because of their thermal, mechanical, and chemical stability $[1,2,3,4]$. Zirconia is an oxygen ion conductor and can serve as an electrolyte in solid oxide fuel cells [5] or as an oxygen sensor [6,7]. Optical applications of zirconia thin films are also under investigation $[8,9]$. Our particular interest in zirconia thin films is the preparation of a model system to obtain better understanding of the acidic properties of sulfated zirconia based materials, which have drawn attention as low temperature isomerization catalysts $[10,11]$.
These applications create a demand for a preparation technique which yields high quality zirconia thin films, i.e. films which are structurally uniform, dense, and adherent to the substrate. A number of techniques are available to prepare oxide films, among them sputtering, vapor deposition, plasma spraying, sol-gel processes (e.g. dip-coating and spin-coating), and electrochemical deposition. However, some of these techniques are expensive and not all of them allow deposition of high quality films on substrates of various compositions and shapes, and under conditions which do not harm the substrate. 
A new technique for the preparation of thin oxide films, referred to as biomimetic synthesis, developed by Bunker et al. [12] uses a self-assembled monolayer (SAM) to mediate film deposition: Bifunctional surfactant molecules $[13,14]$ are attached to a substrate through their surface active head group and a SAM, an ordered array created spontaneously on the substrate through vander Waals interactions between the surfactant's long hydrocarbon chains, is formed [15]. This SAM-coated wafer is immersed into a deposition medium, which contains a dissolved metal salt. Within several hours films consisting of oxides and/or potential oxide precursors such as hydroxides, sulfates, or carbonates (depending on the anions present), deposit on the SAM; concomitantly, in some cases $[16,17,18]$, the formation of a precipitate in the liquid phase is observed. The SAM technique has been successfully applied to prepare adherent films of the following materials on silicon substrates: titania [16,19,20], zirconia [16,17,21], tin oxide [22], iron oxyhydroxide [23,24], and aluminum basic sulfate [25]; and after calcination at temperatures between $473 \mathrm{~K}$ and $873 \mathrm{~K}$ : yttria [16,18], vanadia [26], zinc oxide [27]. Self-assembled monolayers can be patterned using photolithographic techniques $[12,28]$ to create patterned titania films [28] which have potential application as semiconductor devices. In a few cases oriented crystal growth has been observed on selfassembled monolayers or self-assembled multilayers, e.g. in the deposition of zincophosphate zeolite [29], aluminophosphate zeolite [30], or iron oxyhydroxide [31].

De Guire and co-workers [16,17] have previously investigated zirconium-containing films prepared by the SAM technique, focussing on their mechanical stability (adherence), thickness, and microstructure (phase composition). Cross sections of specimens were investigated with transmission electron microscopy (TEM). Samples freshly prepared from zirconium sulfate solutions show a layer consisting of a mixture of tetragonal zirconia and amorphous, presumably sulfate containing material [17]; annealing these samples at 773 $\mathrm{K}$ leads to complete conversion into tetragonal zirconia crystals approx. $10 \mathrm{~nm}$ in diameter which form a fairly dense layer [19]. Film thicknesses (TEM, ellipsometry) up to $180 \mathrm{~nm}$ have been obtained by varying deposition time and conditions [16]. The films were found to be adherent to the substrate using a tape peel test [16].

However, application of zirconia films requires that the film and its surface be structurally uniform not only within a few square nanometers but also on a larger scale. The scope of our work was to elucidate the film morphology with data on the micrometer scale, and to learn at the same time how to optimize the preparation for our purposes. We prepared specimens according to the procedure described in the literature cited above and used scanning electron microscopy (SEM), including energy dispersive X-ray analysis (EDX), and atomic force microscopy (AFM) to investigate all stages of the film preparation.

\section{Experimental}

\section{Preparation}

A detailed description of the preparation of the thin films is given in reference [19]. Pieces $1-3 \mathrm{~cm}^{2}$ of electronicgrade single-crystal (100) silicon wafers $(350 \mu \mathrm{m}$ thick, p-type, polished on one side) were cleaned sequentially with chloroform, acetone, and ethanol; and then were oxidized for 25 min with a $30: 70$ mixture of $30 \% \mathrm{H}_{2} \mathrm{O}_{2}$ and conc. $\mathrm{H}_{2} \mathrm{SO}_{4}$ ('piranha'-solution) at $353 \mathrm{~K}$. The surfactant, ethanethioic acid S-[16(trichlorosilyl)hexadecyl]ester, was prepared following the procedure of Netzer and Sagiv [13]. The SAM was formed by immersing the wafer in a solution of $50 \mu \mathrm{l}$ surfactant in $5 \mathrm{ml}$ bicyclohexyl for 5 hours under argon atmosphere (glove bag). The desired hydrophilic sulfonic acid terminating group is introduced through oxidation of the hydrophobic thioacetate group (a precursor group stable towards reaction with the $-\mathrm{SiCl}_{3}$ group) by immersion into an oversaturated solution of $\mathrm{KHSO}_{5} * \mathrm{KHSO}_{4} * \mathrm{~K}_{2} \mathrm{SO}_{4}$ (Fluka, Deisenhofen, Germany) for 5-17 hours. After this oxidative treatment the wafers were rinsed and immediately transferred into the deposition medium. The deposition of the zirconia films was conducted in a $4 \mathrm{mM}$ solution of zirconium (IV) sulfate tetrahydrate (97\%, Alfa, Karlsruhe, Germany) in $0.4 \mathrm{~N}$ hydrochloric acid. In the standard preparation, the wafers was placed at the bottom of glass vials containing the deposition medium and the top side of the wafer was investigated. Alternatively, the wafer was placed in a sample holder just below the surface of the deposition medium in an upside-down-position and the bottom side was investigated. When the wafer was in the upsidedown-position the deposition medium was sometimes stirred. The vials with wafer and $50 \mathrm{ml}$ of deposition solution were placed into a room temperature oil bath which was then heated to $353 \mathrm{~K}$. Deposition times varied from 4 to 17 hours. Precipitation in the liquid phase was observed (by visual inspection) after approx. $30 \mathrm{~min}$. The wafers were rinsed with distilled water after removal from the deposition medium and were blown dry with argon under ambient conditions. The dried films are designated as 'as-deposited' films. Washing of the wafers was performed by wiping the surface with lintfree tissues (Kimwipes ${ }^{\circledR}$ ) and distilled water. Annealing was performed in air. The wafers were heated at a rate of $5 \mathrm{~K} / \mathrm{min}$ to $773 \mathrm{~K}$ and held at $773 \mathrm{~K}$ for 2 hours.

Analysis

The SEM/EDX analyses were performed with a Hitachi S-4000-FEG / EDAX DX4 using $5 \mathrm{kV}$ acceleration 
voltage and secondary electron mode. AFM images were taken in contact mode with a Burleigh Instruments ARIS 3300 using a small range head ARIS 3005. with a horizontal resolution of $1.0 \mathrm{~nm}$ and a vertical resolution of $10 \mathrm{pm}$. The tip was made of $\mathrm{Si}_{3} \mathrm{~N}_{4}$.

\section{Results}

General observations

Dirt or dust particles were occasionally observed (SEM) on all samples. We ascribe such appearance to the fact that the preparation was not performed in a clean room. Silicon wafers, both as received and after cleaning with solvents, showed smooth, clean surfaces in AFM [this work and 32] as well as SEM pictures. The silicon oxide layer which was formed after oxidation was homogeneous and smooth as analyzed by SEM and AFM, and only silicon and oxygen were detected by EDX. To the unaided eye, SAM-coated wafers had the same appearance as bare silicon wafers, and on the SE micrographs the thioacetate form looked homogeneous and smooth. After oxidative treatment in order to transform the thioacetate terminal groups to sulfonic acid groups, the surface of the wafers showed some structure, indicating either the presence of contaminants or a partial stripping of the SAM, Figure 1.

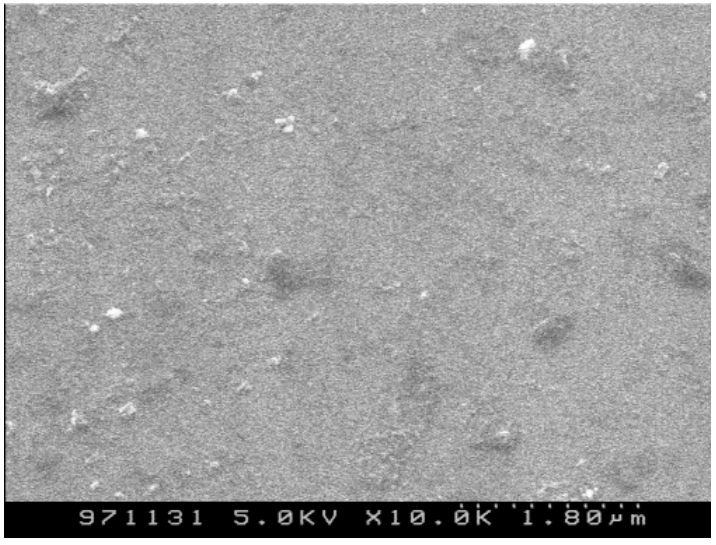

Figure 1: Scanning Electron Micrograph of a selfassembled monolayer on silicon after oxidation of the terminating thioacetate group $(-\mathrm{SCOCH} 3)$ to a sulfonic acid group $(-\mathrm{SO} 3 \mathrm{H})$ with $\mathrm{KHSO} 5 * \mathrm{KHSO} 4 * \mathrm{~K} 2 \mathrm{SO} 4$ for 17 hours at room temperature.

Non-washed as-deposited samples had a shimmering blue and brown appearance. The surface of as-deposited samples showed a distinct morphology (Figures 2-7), decidedly different from the appearance of all previous stages. The formation of a layer-like structure could be recognized. Areas which showed a continuous film and which were not obstructed by deposits consisted of zirconium, sulfur and oxygen (EDX). Silicon was also detected in the analyzed volume; the analyzed depth being ca. $0.35 \mu \mathrm{m}$ at $5 \mathrm{kV}$ excitation energy.

\section{As-deposited films, standard preparation}

SEM images of samples which were rinsed but not washed are shown in Figures 2 and 3. Particles were observed on top of the film surface or partially embedded in the film. The particles seem to be roughly spherical and approximately $0.2-1 \mu \mathrm{m}$ in diameter. Agglomerates of these particles with a size of up to $4 \mu \mathrm{m}$ were also found. The particles contain zirconium, oxygen, and sulfur as the EDX line scan in Figure 2b shows.
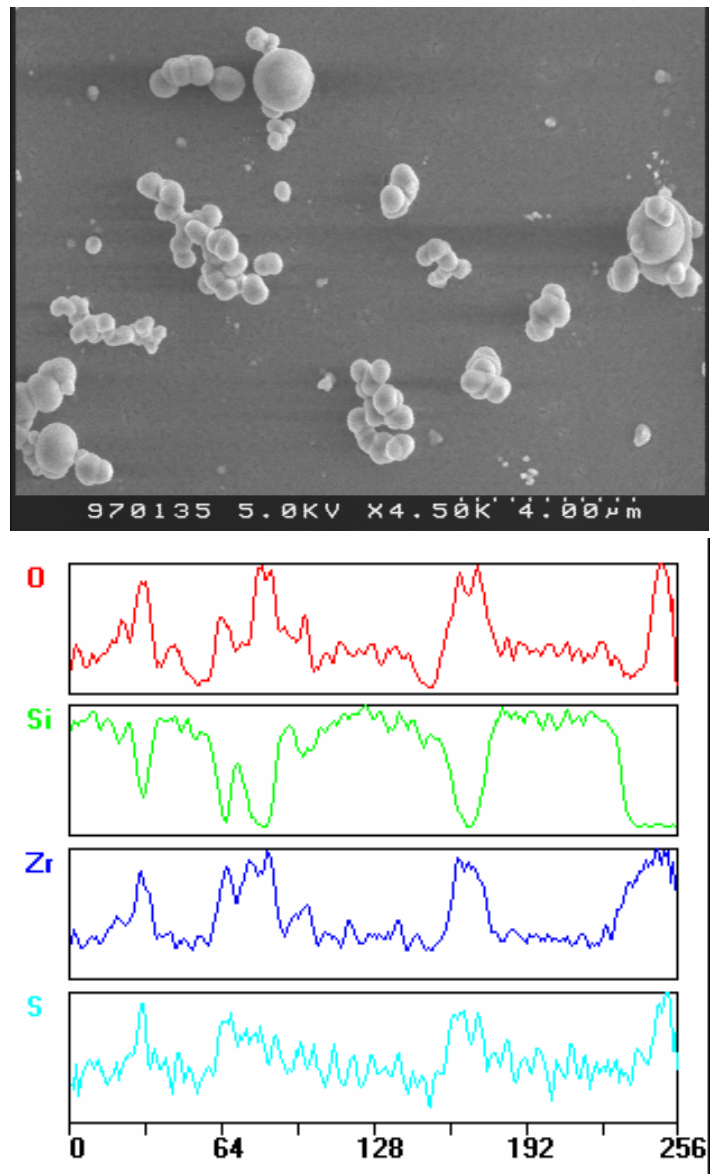

Figure 2: Scanning Electron Micrograph with EDX line scan of a non-washed as-deposited film with precipitate on top of the film. Deposition time: 17 hours.

Consistent with the analyzed depth of approx. $0.35 \mu \mathrm{m}$, the detected silicon concentration is very low in places where particles are on top of the film. Embedded particles are shown in Figures $3 \mathrm{a}$ and $3 \mathrm{~b}$. The particles and their agglomerates could also be detected by AFM, Figure 4. Typical particle diameters obtained from AFM were from 200 to $300 \mathrm{~nm}$, the height above the film was typically $60-120 \mathrm{~nm}$, consistent with partial embedding. Domains where the film was non-continuous were also observed (Figure 5). The extension of such domains was typically several $\mu \mathrm{m}$. These areas appeared to have been incompletely covered, rather than to have lost coverage through film rupture and/or peeling. 

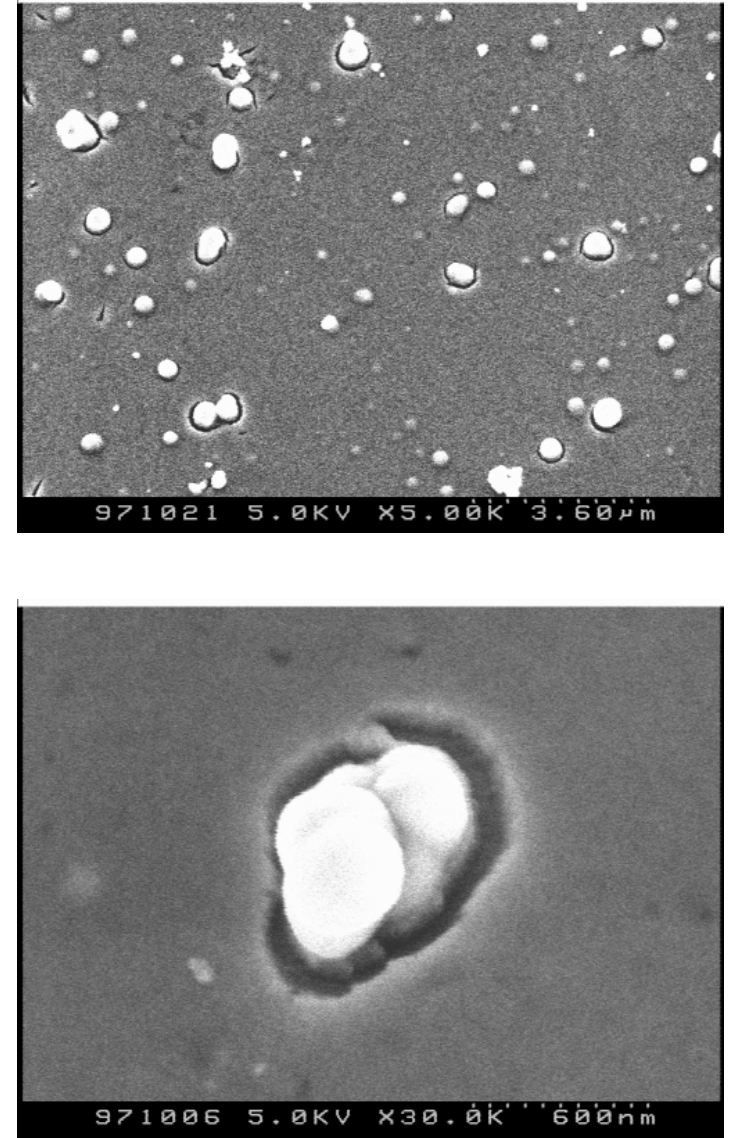

Figures $3 \mathrm{a}$ and $3 \mathrm{~b}$ : Scanning Electron Micrographs of particles embedded into an as-deposited film (nonwashed). Deposition time: 4 hours.

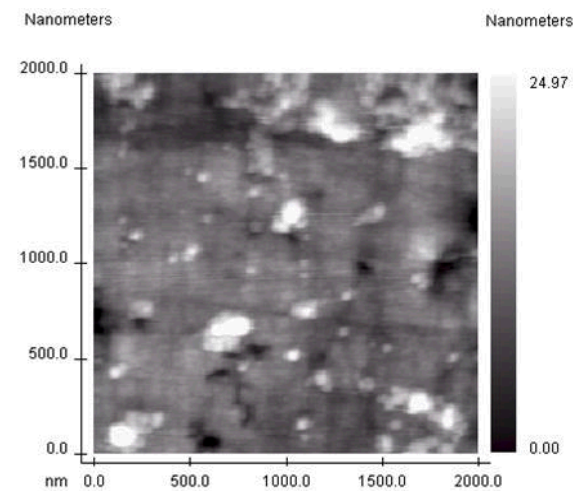

Figure 4: Atomic Force Micrograph of a non-washed asdeposited film. Deposition time: 17 hours.

\section{As-deposited films, upside-down preparation}

For SAM-coated wafers immersed in an upside-down position in an unstirred deposition medium film formation was also observed, indicating that film growth occurs not by sedimentation. The number of particles adhering to the film surface after deposition in the upside-down position was reduced in comparison to the preparation with the wafer at the bottom of the vial, but the adhesion of such particles was not completely prevented.

\section{Washed as-deposited films}

As-deposited films were analyzed after washing with water and a lint-free tissue. Figure 5 shows scanning electron micrographs of the same area on a wafer before (6a) and after (6b) washing. The number of particles on top of the film surface was reduced as a result of washing. Particularly, the number of larger particles (agglomerates) decreased with prolonged and intensive rubbing while smaller particles tended to remain on the film surface. In places where particles were found before washing, the surface was severely damaged after removal of the particles. The defect areas in Figure $6 \mathrm{~b}$ have a size and shape corresponding to the particles in Figure 6a. The large, circle-shaped defects have diameters of typically $0.5-1 \mu \mathrm{m}$ (SEM and AFM) and a depth of at least 30-50 nm (AFM). It could not be unambiguously determined whether these defects were actually holes, with the substrate surface showing through, or whether there was a zirconia film on the bottom of these defects.

Some as-deposited films were characterized by the presence of fissures which could be observed with SEM (Figure 7) and AFM. The fissures were sometimes as long as $1 \mu \mathrm{m}$ and mostly less than $100 \mathrm{~nm}$ wide, sometimes branched, and becoming narrower towards the ends. Opposite edges of such fissures sometimes showed identical curvature, and the edges were often elevated in relation to the film.

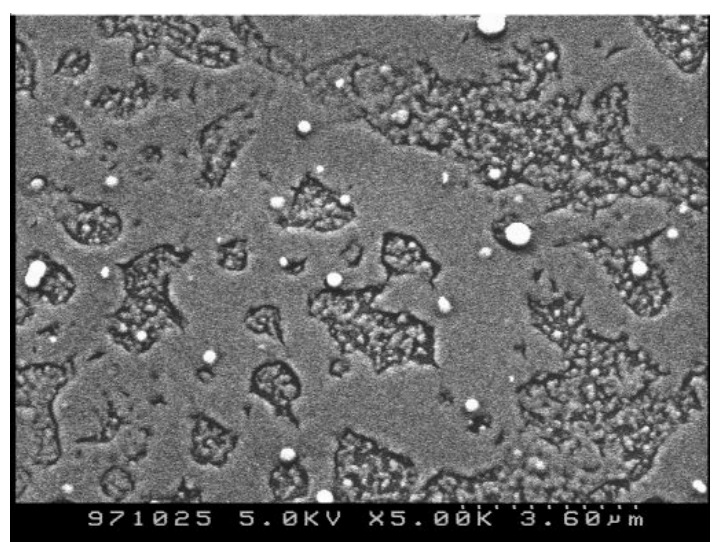

Figure 5: Scanning Electron Micrograph of a nonwashed as-deposited film showing an area with noncontinuous film formation. Deposition time: 4 hours.

Roughness measurements with AFM on as-deposited films, before and after washing, yielded results showing that the films are rather inhomogeneous although areas mainly free of fissures, defects, or adsorbed particles were selected. $R_{r m s}$ varied from 1.4 to $4.6 \mathrm{~nm}$, and $R_{p}$ varied from 6.0 to $22.5 \mathrm{~nm}$. 


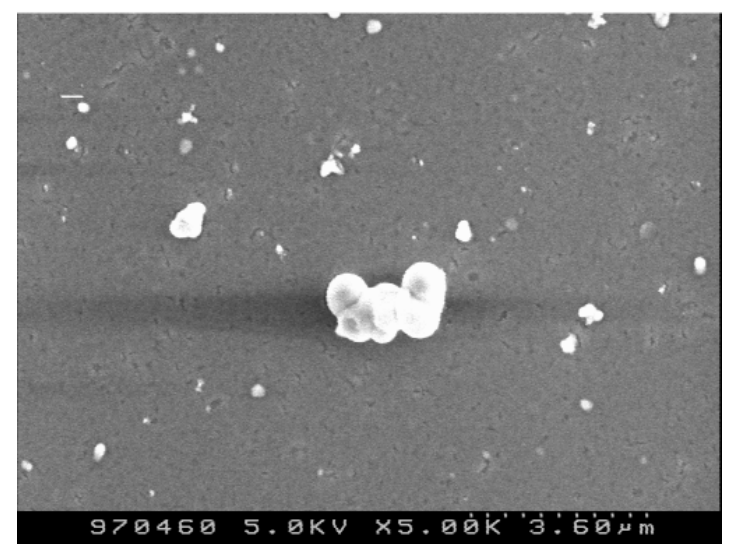

Figure 6a: Scanning Electron Micrograph of an asdeposited film, before washing. Deposition time: 17 hours.

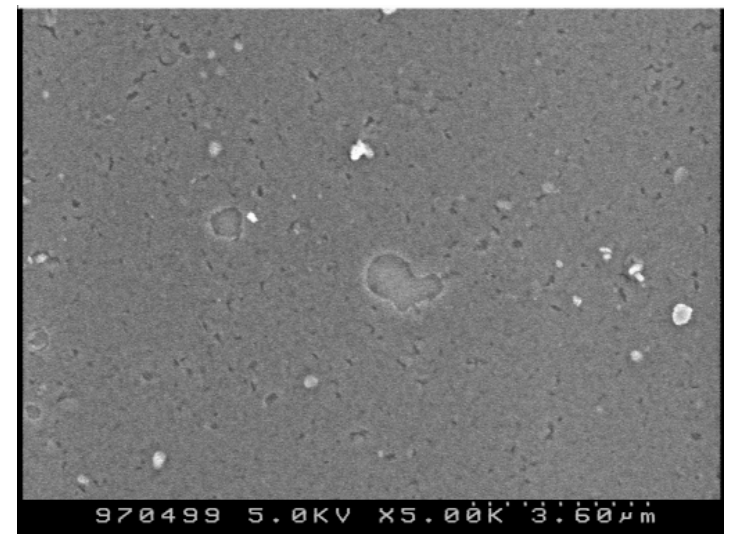

Figure 6b: Scanning Electron Micrograph of the same area of the film as in 5a after washing the sample with distilled water and a tissue.

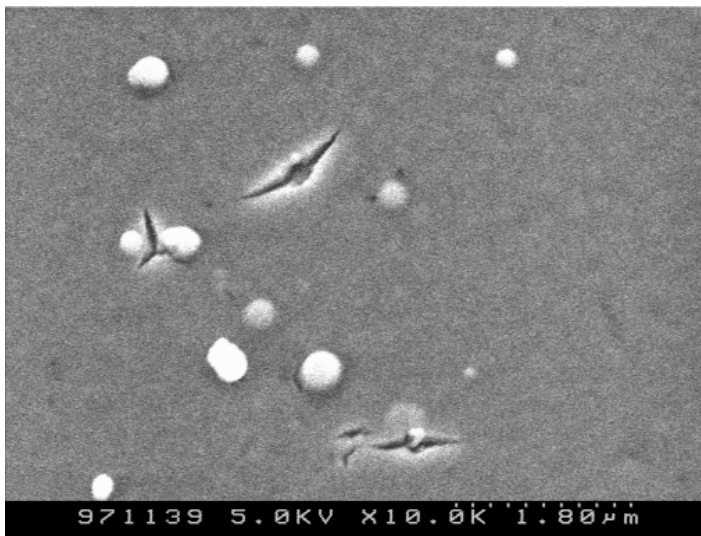

Figure 7: Scanning Electron Micrograph of fissures in a non-washed as-deposited film washed. Deposition time: 16 hours.

\section{Calcined films}

Pictures of films calcined at $773 \mathrm{~K}$ in air are given in Figures 8 and 9. The SEM image in Fig. 8 shows a typical circle-shaped defect at the right border; otherwise the film is characterized by a number of small fissures which were already visible on the uncalcined film. The AFM image in Fig. 9 shows some small adhering precipitate particles, and several defects in the film, i.e. a few small spots and one large patch. It could not be identified if fissures - when present - widened or their number increased during calcination. Calcined films were smoother than as-deposited films, with typical $\mathrm{R}_{\mathrm{rms}}=$ $0.7-0.8 \mathrm{~nm}$ and typical $\mathrm{R}_{\mathrm{p}}=3.5-3.9 \mathrm{~nm}$. The films appear to still be adherent to the substrate surface after calcination.

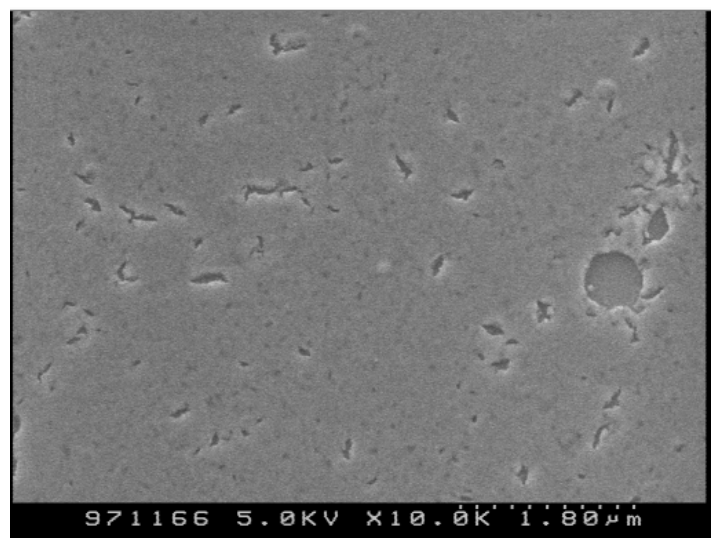

Figure 8: Scanning Electron Micrograph of a washed and calcined film with circular defect and fissures. Deposition time: 4 hours; calcination: 2 hours at $773 \mathrm{~K}$, air.

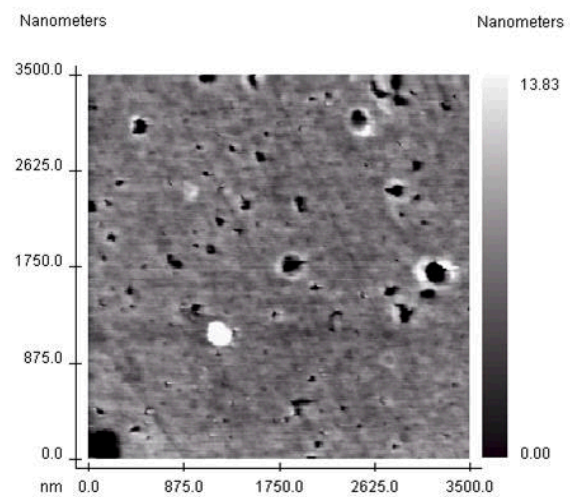

Figure 9: Atomic Force Micrograph of a washed and calcined film. Deposition time: 4 hours; calcination: 2 hours at $773 \mathrm{~K}$, air.

\section{Discussion}

Morphology of as-deposited films, relation to literature Agarwal et al. [17] and De Guire et al. [16] investigated zirconium-containing films deposited on SAMs from aqueous medium. The thickness of the films as measured with TEM and ellipsometry varied from 45-180 nm depending on the deposition conditions. From TEM/EDX and XPS data it was concluded that these 
films in their as-deposited state consist of amorphous basic zirconium sulphate plus 3-6 nm crystallites of tetragonal zirconia. The microstructure of the films varied in the direction normal to the surface [33]: the crystallite size decreased and the fraction of amorphous material increased with distance from the substrate. The films adhered so well to the substrate that in a tape peel test no deposit on the tape was found with XPS [16]. Calcination at $773 \mathrm{~K}$ for 2 hours led to complete conversion into tetragonal zirconia with maximum crystallite sizes of $10 \mathrm{~nm}$ [19] without disruption of the film [17].

SEM analysis of our as-deposited samples show that most of the wafer is covered by a film with characteristics decidedly different from the features of the substrate. EDX analysis indicates the presence of zirconium, oxygen, and sulfur in areas with continuous film formation. Sulfur has to be assigned to the film, because the sulfur of the SAM was not detectable with EDX. The elemental analysis is consistent with the phases identified by De Guire [16], i.e. zirconia and a basic zirconium sulfate. The film looks rather flat and smooth and, consistent with the nanocrystallinity of such films [16,17], it was not possible to resolve substructures with SEM. AFM investigations yielded an roughnesses $R_{p}$ in the range of 6.0 to $22.5 \mathrm{~nm}$ in areas of the asdeposited films without adsorbed particles, fissures, or damaged areas, which is consistent with the picture of a surface composed of nano-sized particles [32]. Although the SAM was apparently imperfect prior to deposition, continuous film formation dominated, indicating that minor defects may be overgrown, but the presence of areas with non-continuous film formation (Figure 5) may indicate that contaminants or major defects in the SAM prohibit film growth, consistent with the fact that a SAM has been inferred to be necessary for the formation of a continuous and adherent film [17].

\section{Adsorbed particles and effect of washing}

In addition to the film, roughly spherical particles $0.2-1$ $\mu \mathrm{m}$ in diameter and large agglomerates of these particles were found on top of the film and sometimes also partially embedded in the film. SEM and AFM gave consistent results as to the shape and size of these particles which were composed of zirconium, oxygen and sulfur, similar to the film composition. However, this composition is also consistent with the composition of the precipitate formed in the liquid phase during deposition which was identified by De Guire [16], using infrared spectroscopy, thermogravimetry, and EDX, as $\mathrm{Zr}_{2}(\mathrm{OH})_{6} \mathrm{SO}_{4} * 2 \mathrm{H}_{2} \mathrm{O}$. The adsorbed particles are similar in their appearance to a particulate precipitate that Shi and Gao [34] obtained by homogeneous precipitation from a solution containing zirconium ions, sulfate, and urea, and which consisted of equisized spheres. The composition of the precipitate was analyzed to be either $\mathrm{Zr}_{2}\left(\mathrm{SO}_{4}\right)(\mathrm{OH})_{6} * 6 \mathrm{H}_{2} \mathrm{O}$ or $\mathrm{Zr}_{2} \mathrm{O}_{2}(\mathrm{OH})_{2}\left(\mathrm{SO}_{4}\right) * 6 \mathrm{H}_{2} \mathrm{O}$ [34], similar to De Guire's analysis [16].

The $200 \mathrm{~nm}$ and larger primary particle size of the adsorbate exceeds the size of the crystals forming the film by one order of magnitude, indicating that these particles originate from earlier nucleation events or have grown faster than the film particles. The agglomerates of these particles are large enough to interact with visible light, consistent with the observation of a milky reaction medium and subsequent visible precipitation. We infer that the adsorbed particles are the same species as the precipitate in the liquid phase, and that this bulk precipitate adheres to the surface during (embedded particles) and after film formation. Depending on their size and/or degree of embedding, particles adhering to our oxidic zirconium films can be washed off more or less easily by wiping the surface with tissues and water. Unfortunately, although they can be removed, these particles leave defects behind, typically patches up to 0.5 $\mu \mathrm{m}$ in diameter where the film or the film surface has been pulled off. Attempts to avoid the adhesion of such particles and thus the number of such defects by modifying the deposition geometry were only partially successful. The bottom sides of wafers suspended in an unstirred deposition medium showed a film with fewer adsorbed precipitate particles in comparison to the standard preparation method, but the number was still significant. The flaws in the film may interfere with application of the films as protective coatings, and makes them unsuitable as model catalysts. It would appear that an easy way to resolve such particle adhesion would be to work with stable deposition solutions. However, it has been inferred that the reaction medium needs to be unstable towards colloid formation and bulk precipitation during the deposition to grow zirconia films thicker than $3 \mathrm{~nm}$ [17]. The dilemma which arises from these findings raises the question whether the mechanism of film formation requires such unstable zirconium salt solutions.

\section{Film Growth Mechanism}

Two different mechanisms are discussed in the literature $[35,36]$ for film deposition from liquid phase: (i) cluster growth whereby particles formed in the liquid phase (homogeneous nucleation) adsorb on the SAM and coagulate to form a film (ii) ion-by-ion growth whereby anions and cations adsorb successively on the substrate (heterogeneous nucleation). The two mechanisms may sometimes occur simultaneously [35].

Selection of deposition conditions can direct the growth mechanism. Bunker et al. [12] investigated the deposition of calcium oxalate and identified the level of supersaturation and the net solution-particle interfacial energy as key parameters: At low levels of 
supersaturation only few surfaces induce heterogeneous nucleation. At high levels of supersaturation, nucleation occurs on most surfaces independent of the solutionparticle interfacial energy, and growth kinetics are enhanced. In a successful biomimetic synthesis [12] heterogeneous nucleation is promoted and homogeneous nucleation is suppressed. Suppression of homogeneous nucleation excludes the cluster growth mechanism.

The properties of the film correlate with the deposition mechanism. Rieke and Bentjen [37] deposited CdS on silicon, and described the mechanism as a burst type nucleation confined to the substrate surface with subsequent crystal growth, leading to films with monodispersed crystallite size. Bulk precipitation was observed at longer deposition times but film formation was not attributed to adherence of clusters. Similar to our observations, the authors found adsorption of bulk precipitate on top of the CdS films, and their micrographs show such adsorbed particles even when the reaction medium did not yet visibly precipitate. Gorer et al. [38] prepared $\mathrm{PbSe}$ films on glass from solutions which additionally contained a complexant at various concentrations. High complexant-to-metal ratios were linked to an ion-by-ion deposition mechanism and the resulting patchy films consisted of aggregates of 8-15 nm crystals. Low complexant-to-metal ratios led to a cluster deposition mechanism and the films appeared homogeneous, consisting typically of $4 \mathrm{~nm}$ crystallites with less than $10 \%$ of larger crystallites. Meldrum et al. [36] investigated the chemical deposition of $\mathrm{PbS}$ on SAMs finding a homogeneous crystal size distribution after rapid depositions with bulk precipitation, indicating that the nuclei were produced in a single nucleation event [39]; a larger size distribution was found after slow deposition, suggesting further nucleation after the initial event. The data were interpreted in terms of an ion-byion growth mechanism, i.e. nucleation on the substrate followed by particle growth. It is also stated in the literature that the observation of epitaxial growth presents strong evidence for an ion-by-ion mechanism [35]. It seems that the mechanism can be directed either towards true biomimetic processing which results in slow, possibly oriented, growth, larger particles, and larger particle size distributions; or towards homogeneous nucleation which results in rapid unoriented growth, and narrow particle size distributions. In this context it should be mentioned that the observation of a bulk precipitate is only a delayed indicator for homogeneous nucleation because precipitate becomes visible only after nucleation has been followed by substantial particle growth.

Zirconia films prepared by the SAM approach are believed to grow following a homogeneous nucleation process. Agarwal et al. [17] and Shin et al. [40], having observed neither substantial film growth in clear solutions nor any orientation of the nanocrystals, but having observed a chemical, crystallographical and microstructural similarity between the films and the precipitate formed in the liquid phase, explained the mechanism of film formation and the growth kinetics through a coagulation model. The driving forces for film formation, i.e., attractive interactions between the SAM and colloidal particles in the liquid phase could be described using DLVO theory [40]. Consistent with the idea of cluster growth in this particular case is the fact that certain ions such as sulfate and phosphate are known to promote polymerization in the liquid phase by forming bridging structures to yield polynuclear metal complexes [39] which could be precursor structures.

We have demonstrated that our oxidic zirconium films are not formed by sedimentation; indicating attractive interactions between the SAM and either clusters or ions in the liquid phase. We have also shown that working under conditions where homogeneous nucleation occurs has a drawback: the particles of the precipitate in the liquid phase are incorporated into the film or adsorbed on the film surface, and removal of these particles, if possible, severely damages the film. The presence of such defects is not specific to zirconia films; titania films have shown similar features [32] although they were prepared from a deposition medium which stayed clear to the eye. The features on the titania films are described as protrusions and depressions of less than $25 \mathrm{~nm}$ height/depth and less than $200 \mathrm{~nm}$ width. Such defects are inherent to the method as long as uncontrolled particle growth in the deposition medium may start anytime. Promising results were obtained with solutions clear to the eye, in 12-48 $\mathrm{h}$ lasting depositions at lower temperatures [41] as well as with a sequence of very short depositions [32] at $343 \mathrm{~K}$.

\section{Fissure formation}

Fissures were observed in some as-deposited and calcined films. Fissures, as shown in Figure 7, are identified as cracks based on the identical curvature of opposite edges. Figure 7 also seems to indicate that fissures are more frequent in places where particles are (or were) adsorbed. Rupture of the film could be caused by volume changes during drying and additionally during annealing. It has been reported in the literature that the film thickness is reduced by $40 \%$ after annealing at 773 $\mathrm{K}$ [17], and dehydration and shrinkage are already observed after treatment at $473 \mathrm{~K}$ [19]. The amorphous fraction of the film contains water [19], however, the volume change during drying at room temperature may be small. Possible further dehydration during evacuation for SEM does not induce major changes because the cracks were also observed with AFM under ambient conditions. Another cause of cracks, the stress induced by different thermal expansion of substrate and film, 
either during cooling after the deposition or during annealing, may partially be mitigated by the SAM or its decomposition products. Pyrolysis of the SAM occurs at 473-673 K in air, apparently without disruption of adherence or continuity of the film [16].

\section{Comparison with other preparation techniques}

Zirconia films on aluminum prepared by Connor et al. [42] according to the sol-gel technique show cracks which are about $1 \mu \mathrm{m}$ wide and more than $10 \mu \mathrm{m}$ long with the film being lifted off of the substrate surface along the cracks. Di Maggio et al. [3] used dip-coating to prepare mixed ceria-zirconia films on stainless steel, and obtained a coating with a granular surface after annealing in vacuum, while thermal treatment in air yielded a visibly cracked coating. Quinson et al. [4] prepared zirconia films on steel by dip-coating. After annealing for $5 \mathrm{~min}$. at $773 \mathrm{~K}$, the films were elastic and adherent but too porous to inhibit corrosion of the steel. The surfaces of yttria-stabilized zirconia films were crack-free but grainy after treatment at $1323 \mathrm{~K}$ [1]. Atik et al. [2] prepared zirconia films through a sol-gel process involving acid-sonocatalysis. Treated at $1073 \mathrm{~K}$ in air these films showed cracks several $\mu \mathrm{m}$ long and up to 0.5 $\mu \mathrm{m}$ wide when heated rapidly, and they were crack-free but grainy ( $\mu \mathrm{m}$ scale) when heated slowly. Our films appear to have about one order of magnitude smaller cracks, do not peel off, and are less grainy than the films in the above references; however, the different treatments allow only a limited comparison.

\section{Conclusions}

Thin oxidic films of zirconium were deposited on selfassembled monolayers by immersing a SAM-coated Si-

\section{References}

wafer into a zirconium salt solution. Bulk (visible) precipitation, according to the literature [17] a prerequisite for the formation of films thicker than $3 \mathrm{~nm}$, occurs in the liquid phase during the deposition. The films were investigated by SEM and AFM and the following characteristics were identified:

The presence of particles which are adsorbed on top and embedded in the film could be linked to the presence of bulk precipitate in the liquid phase. These spherically shaped particles which are ca. $200 \mathrm{~nm}$ large and sometimes agglomerated, can be washed off of the film with water and tissues but the film or at least the film surface is pulled off in these places. The preparation in a deposition medium with homogeneous nucleation followed by precipitation is thus disadvantageous because it leads to defects in the film. Fissures in the film surface, less than $100 \mathrm{~nm}$ wide and several $100 \mathrm{~nm}$ long, were observed; they are most likely a result of shrinkage of the film during drying.

Scanning electron microscopy (SEM/EDX) together with atomic force microscopy (AFM) proved to be excellent to investigate the morphology and composition of chemically deposited thin films. Specifically, SEM allows fast characterization on the scale of several $\mu \mathrm{ms}$, yielding information pertinent to many applications of such films. Characterizations performed previously give incomplete pictures because they either lacked spatial resolution (XPS) or because only an extremely small area was analyzed (TEM)

\section{Acknowledgements}

E. Kitzelmann is thanked for her assistance with the AFM data acquisition.

1. M. Shane and M.L. Mecartney, J. Mater. Sci. 25, 1537 (1990)

2. M. Atik, J. Zarzycki, and C. R'Kha, J. Mater. Sci. Lett. 13, 266 (1994).

3. R. Di Maggio, L. Fedrizzi, S. Rossi, and P. Scardi, Thin Solid Films 286, 127 (1996).

4. J.F. Quinson, C. Chino, A.M. Becdelievre, C. Guizard, and M. Brunel, J. Mater. Sci. 31, 5179 (1996).

5. N.J. Maskalick and C.C. Sun, J. Electrochem. Soc. 118, 1386 (1971).

6. M. Croset, P. Schnell, and G. Valesco, J. Vac. Sci. Technol. 14, 777 (1977).

7. Y. Miyahara, K. Tsukada, and H. Miyagi, J. Appl. Phys. 63, 2431 (1988).

8. K.V.S.R. Apparao, N.K. Sahoo, and T.C. Bagchi, Thin Solid Films 129, L71 (1985).

9. A. Duparré, E. Welsch, H.-G. Walther, N. Kaiser, H. Müller, E. Hacker, H. Lauth, J. Meyer, and P. Weissbrodt, Thin Solid Films 187, 275 (1990).

10. M. Hino, S. Kobayashi, and K. Arata, J. Am. Chem. Soc. 101, 6439 (1979).

11. X. Song and A. Sayari, Catal. Rev. - Sci. Engr. 38, 329 (1996).

12. B.C. Bunker, P.C. Rieke, B.J. Tarasevich, A.A. Campbell, G.E. Fryxell, G.L. Graff, L. Song, J. Liu, J.W. Virden, and G.L. McVay, Science 264, 48 (1994).

13. L. Netzer and J. Sagiv, J. Am. Chem. Soc. 105, 674 (1983).

14. R. Collins and C.N. Sukenik, Langmuir 11, 2322 (1995).

15. A. Ulman, Chem. Rev. 96, 1533 (1996).

16. M.R. DeGuire, H. Shin, R. Collins, M. Agarwal, C.N. Sukenik, and A. Heuer, Proc. SPIE 2686, 88 (1996).

17. M. Agarwal, M.R. De Guire, and A.H. Heuer, J. Am. Ceram. Soc. 80, 2967 (1997).

18. M. Agarwal, M.R. De Guire, and A.H. Heuer, Appl. Phys. Lett. 71, 891 (1997) 
19. H. Shin, M. Agarwal, M.R. De Guire, and A. Heuer, J. Am. Ceram. Soc. 79, 1975 (1996).

20. H. Shin, R.J. Collins, M.R. De Guire, A.H. Heuer, and C.N. Sukenik, J. Mater. Res. 10, 692 (1995)

21. M. Agarwal, Dissertation, Case Western Reserve University, Cleveland, Ohio (1996).

22. S. Supothina and M.R. De Guire, submitted to Thin Solid Films.

23. P.C. Rieke, B.D. Marsh, L.L. Wood, B.J. Tarasevich, J. Liu, L. Song, and G.E. Fryxell, Langmuir 11, 318 (1995).

24. P.C. Rieke, R. Wiecek, B.D. Marsh, L.L. Wood, J. Liu, L. Song, G.E. Fryxell, and B.J. Tarasevich, Langmuir 12, 4266 (1996).

25. Y. Wang, S. Supothina, M.R. De Guire, A.H. Heuer, R.J. Collins, and C.N. Sukenik, Chem. Mater. 10, $2135-44$ (1998).

26. T.P. Niesen, J. Wolff, J. Bill, T. Wagner, and F. Aldinger, submitted to Proc. $9^{\text {th }}$ CIMTEC, 14-19 June 1998, Florence, Italy.

27. M.R. De Guire, T.P. Niesen, J. Wolff, S. Supothina, J. Bill, F. Aldinger, and M. Rühle, Proc. Workshop on Grain Boundary Dynamics of Precursor-Derived Covalent Ceramics, Schloss Ringberg, Germany, 10-14 November, 1996. F. Aldinger and J. Bill, eds. Wiley-VCH Verlagsgesellschaft $\mathrm{mbH}$ (accepted for publication)

28. R.J. Collins, H. Shin, M.R. De Guire, A.H. Heuer, and C. Sukenik, Appl. Phys. Lett. 69, 860 (1996).

29. S. Feng and T. Bein, Nature 368, 834 (1994).

30. S. Feng and T. Bein, Science 265, 1839 (1994).

31. B.J. Tarasevich, P.C. Rieke, and J. Liu, Chem. Mater. 8, 292 (1996).

32. T.P. Niesen, M.R. De Guire, J. Bill, F. Aldinger, M. Rühle, A. Fischer, F.C. Jentoft, and R. Schlögl, submitted to J. Mater. Res.

33. M. Agarwal, M.R. De Guire, and A.H. Heuer, Appl. Phys. Lett. 71, 891 (1997)

34. J.L. Shi and J.H. Gao, J. Mater. Sci. 30, 793 (1995).

35. S. Gorer and G. Hodes, J. Phys. Chem. 98, 5338 (1994).

36. F.C. Meldrum, J. Flath, and W. Knoll, Langmuir 13, 2033 (1997).

37. P.C. Rieke and S.B. Bentjen, Chem. Mater. 5, 43 (1993).

38. S. Gorer, A. Albu-Yaron, and G. Hodes, Chem. Mater. 7, 1243 (1995).

39. E. Matijevic, Acc. Chem. Res. 14, 22 (1981).

40. H. Shin, M. Agarwal, M.R. De Guire, and A.H. Heuer, Acta mater. 46, 801 (1998).

41. A. Fischer, F.C. Jentoft, and R. Schlögl, unpublished results.

42. P.A. Connor, K.D. Dobson, and A.J. Mcquillan, Langmuir 11, 4193 (1995). 